\title{
Broadband acoustooptic diffraction of two-wavelength light in paratellurite
}

\author{
Pilgun Yu.V. and Smirnov Ye.M. \\ National Taras Shevchenko University of Kyiv, 64 Volodymyrska St., \\ 01601 Kyiv, Ukraine, e-mail: ply@univ.kiev.ua
}

Received: 28.04.2009

After revision: 09.12.2009

\begin{abstract}
We suggest acoustooptic deflector operating at two different light wavelengths. Geometry of paratellurite crystal is chosen such that it simultaneously satisfies the Bragg conditions at those wavelengths. This enables beam steering at either of the wavelengths in the same predefined angular range. The wavelengths are switched by means of changing the sound frequency, so that only one of the wavelengths is available at the output of the deflector at each specific moment of time. A technique for calculations of parameters of the device is described. The deflector has been successfully constructed and investigated experimentally.
\end{abstract}

Keywords: acoustooptic diffraction, two-wavelength light, deflectors, dualwavelength operation, paratellurite

PACS: $78.20 . \mathrm{Hp}$, 42.79.Jq

UDC: $535.42,534.522 .1$

\section{Introduction}

Acoustooptic devices are the best choice for applications requiring spatial scanning of laser beams. Acoustooptic deflectors have many advantages over electro-mechanical devices, such as fast response time, high precision, and stability of a raster obtained. In the fields of laser scanning microscopy and profilometry, measurements of surfaces of specimens are usually performed over some predefined grid of points. In order to resolve height ambiguity, there is a need in performing investigations of a given object at different light wavelengths and at the same scanning points. For such a task one needs a device capable of performing beam scanning and switching between the wavelengths.

Several constructions of bichromatic acoustooptic devices are already known, though many of them have a number of limitations. Bichromatic deflector presented in the work [1] requires additional correction lenses to superimpose rasters of different wavelengths. Moreover, the above device performs simultaneous scanning of beams at both wavelengths, though a sequential scanning is often needed. In another work [2], a bichromatic light modulator has been described that uses a prism for recombining beams of different wavelengths after diffraction. An acoustooptic device capable of wavelength switching has been considered in the study [3], however it does not perform scanning of 
beams. Finally, all of these devices do not allow for scanning of objects under test with the beam at one light wavelength at first and at a second wavelength afterwards.

Below we will develop an acoustooptic deflector that operates at two wavelengths of light and also acts as a wavelength-switching device. A similar device enabling independent control of two wavelengths has been described in the recent study [4]. However, no calculation details have been given there, so that justification of the cell geometry selected by the authors still remains not obvious and, moreover, the control of different wavelengths has been performed by separate ultrasonic transducers, which complicates both construction and driving electronics of the device. In particular, in the current work we analyse possibilities for satisfying the Bragg conditions at two wavelengths of light and for using a single transducer to control them. The calculations are presented for the case of deflector made on paratellurite $\left(\mathrm{TeO}_{2}\right)$ and operating at the wavelengths of 632.8 and $785.0 \mathrm{~nm}$.

\section{Broadband acoustooptic diffraction}

Usually acoustooptic deflectors are designed for operating at a single light wavelength. The geometry of acoustooptic interaction depends on the wavelength, so any changes in the working wavelength requires changing incident and deflecting angles. The incident angle could be adjusted mechanically but this is not desirable. For the case of twowavelength operation we need a device, for which no mechanical readjusting is involved. Unique properties of paratellurite allow constructing very efficient deflectors requiring relatively low ultrasound power. The most used configuration is based on the off-axis anisotropic Bragg diffraction [5]. A relevant geometry of acoustooptic interaction is depicted in Fig. 1.

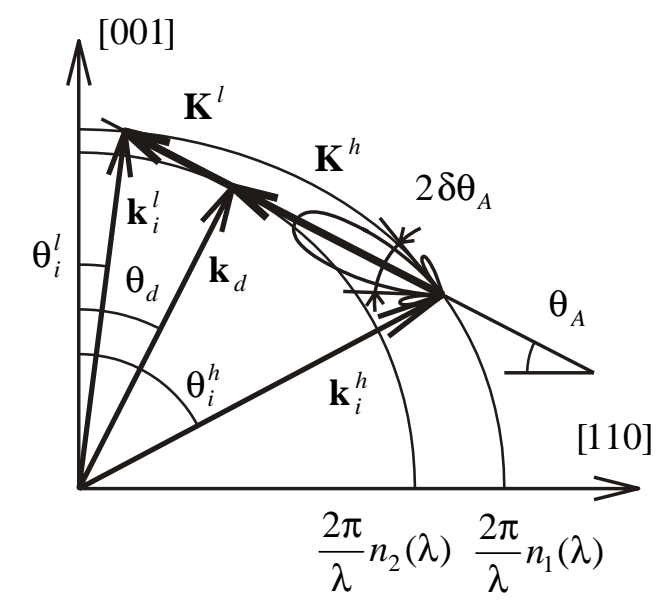

Fig. 1. Geometry of anisotropic interaction in the paratellurite. Lower-frequency $\left(\mathbf{K}^{l}\right)$ and higher-frequency $\left(\mathbf{K}^{h}\right)$ cases require different incident angles $\theta_{i}^{l}$ and $\theta_{i}^{h}$, though they result in the same diffraction angle $\theta_{d}$.

An input light (the wave vector $\mathbf{k}_{i}$ ) interacts with a sound wave ( $\left.\mathbf{K}\right)$ and produces a diffracted wave $\left(\mathbf{k}_{d}\right)$. For a given direction of sound propagation there are two possibilities for satisfying the momentum-matching condition. In the first case the wave vector equation is given by $\mathbf{k}_{d}=\mathbf{k}_{i}^{h}+\mathbf{K}^{h}$ and then the optical frequency is shifted up 
$\left(\omega_{d}=\omega_{i}+\Omega\right)$ to satisfy conservation of energy. In the second case the wave vector relation reads as $\mathbf{k}_{d}=\mathbf{k}_{i}^{l}-\mathbf{K}^{l}$ and the optical frequency is down-shifted $\left(\omega_{d}=\omega_{i}-\Omega\right)$. The first configuration requires higher sound frequency due to the inequality $\left|\mathbf{K}^{h}\right|>\left|\mathbf{K}^{l}\right|$. The both cases are possible at the same time, but only one is realised in the device by proper orientation of the input light direction. We will distinguish between those versions of device, which use higher and lower frequencies of sound, by labelling the variables with a corresponding superscript (e.g., $\mathbf{K}^{h}$ and $\mathbf{K}^{l}$ ). Note than if we reverse direction of the sound propagation, the optical frequency shift will change its sign but general characteristics of the interaction will remain the same.

The lengths of the wave vectors $\mathbf{k}_{i}^{h}, \mathbf{k}_{i}^{l}$ and $\mathbf{k}_{d}$ are constrained by angular dependences of the refractive indices for ordinary and extraordinary waves in paratellurite. When dealing with the construction proposed by us, the incident light corresponds to the extraordinary wave and the diffracted light to the ordinary one. Optical activity of the paratellurite manifests itself when the light propagates very close to the optic axis [001], so that we could assume the light to remain linearly polarised. The direction of the incident light should remain fixed and, therefore, we should decide which of the possible angular values, $\theta_{i}^{h}$ or $\theta_{i}^{l}$, will be actual for the device. Usually the smaller angle $\theta_{i}^{l}$ is preferred because it corresponds to lower ultrasound frequency.

The diffraction angle $\theta_{d}$ may be controlled by changing the sound frequency $\Omega$. The sound wave vector $\mathbf{K}$ is selected to be tangential to the optical wave-normal surface $2 \pi n_{2}(\theta) / \lambda$, so that even great changes in the $\mathbf{K}$ length would cause only small deviation from the exact momentum-matching condition. The remaining discrepancy is compensated by inevitable divergence of the sound wave. Therefore the angle of the sound beam divergence $\delta \theta_{A}$ would determine the range of available light diffraction angles.

Typical construction of our acoustooptic cell made on the basis of paratellurite is shown in Fig. 2. The phase velocity of the sound wave is normal to the plane of transducer and directed at the angle $\theta_{A}$ with respect to crystallographic direction [110]. The

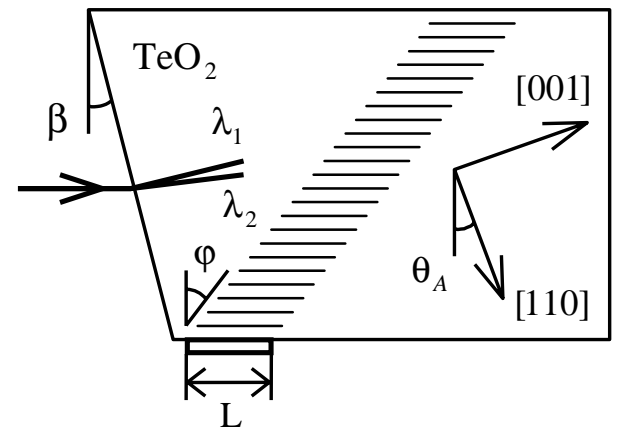

Fig. 2. Sketch of our acoustooptic cell. sound energy flow vector does not coincide with the corresponding wave vector, due to high acoustic anisotropy present in the paratellurite crystals. The deviation angle $\varphi$ of the group velocity is approximately equal to $50^{\circ}$ if the sound propagation angle $\theta_{A}$ is about $8^{\circ}$. The size $L \times H$ of the transducer would determine angular divergence of the sound beam. 
Possible geometries of the acoustooptic interactions for two different light wavelengths are illustrated in Fig. 3. The interaction geometries differ for different wavelengths $\lambda_{1}$ and $\lambda_{2}$. Both the scale and the shape of the wave-normal surfaces vary, due to dispersion. In the present work we use the same transducer for the both wavelengths and so the direction of the sound propagation is the same.

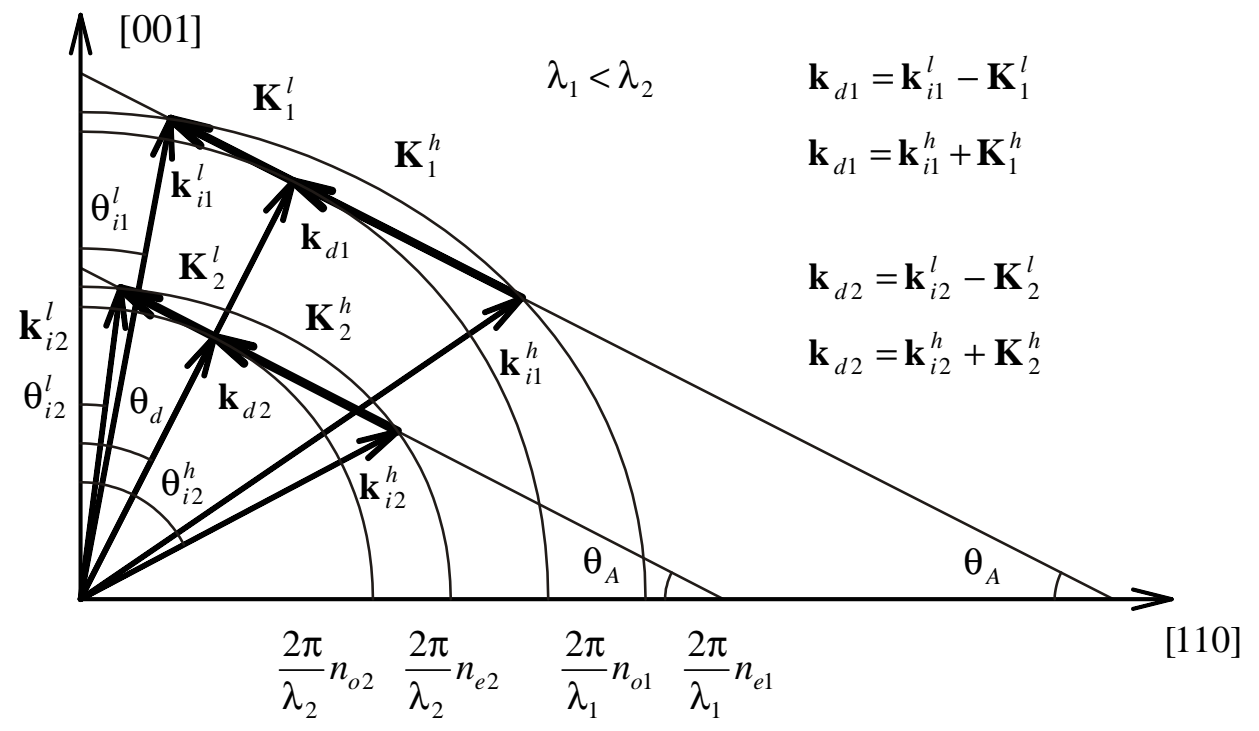

Fig. 3. Acoustooptic interactions for two different light wavelengths

To enable dual-wavelength operation, the Bragg condition should be satisfied at the both light wavelengths simultaneously and therefore different angles of incidence $\theta_{i 1}$ and $\theta_{i 2}$ are required for the first and the second wavelengths, respectively. In order to obtain the required incident angles, the input laser beams should have different directions. However, this would complicate the construction and need additional tuning of the device. As another option, a high-dispersive prism might be placed for separating different wavelengths of collinear laser beams. The crystal of paratellurite itself could act as such a prism if its input face is inclined. Usually the crystal already has prismatic shape to make incoming and diffracted light beams parallel. A prismatic construction has another advantage: a zero-order diffracted beam leaves the acoustooptic cell in off-axis manner and the latter beam may be easily blocked by a diaphragm. Inclination of the input face may also be used to enable two-wavelength operation of the deflector. Incident laser beams are refracted on the input crystal face and the light of different wavelengths is refracted at different angles, again due to dispersion in the paratellurite. This is why, if the inclination angle $\beta$ of the input face is chosen properly, it is possible to make refracted beams propagate inside the crystal at angles needed for efficient Bragg diffraction at the both wavelengths simultaneously. In the current work we just intend to examine how the construction angle $\beta$ and the internal angles of interaction should be chosen for constructing a two-wavelength deflector. 


\section{Refractive indices of paratellurite}

The paratellurite represents optically uniaxial crystal possessing optical activity and belongs to the point symmetry group 422 . The refractive indices of the ordinary and extraordinary rays in such crystals may be found [6] from the relation

$$
\left(n^{2}-n_{10}^{2}\right)\left(n^{2}-n_{20}^{2}\right)=G^{2},
$$

where $n_{10}$ and $n_{20}$ denote the refractive indices respectively for extraordinary and ordinary rays determined as if there were no optical activity, and $G$ is the gyration coefficient. Solving Eq. (1) results in

$$
\begin{aligned}
& n_{1}=\frac{1}{\sqrt{2}} \sqrt{n_{10}^{2}+n_{20}^{2}+\sqrt{\left(n_{20}^{2}-n_{10}^{2}\right)^{2}+4 G^{2}}}, \\
& n_{2}=\frac{1}{\sqrt{2}} \sqrt{n_{10}^{2}+n_{20}^{2}-\sqrt{\left(n_{20}^{2}-n_{10}^{2}\right)^{2}+4 G^{2}}},
\end{aligned}
$$

with $n_{1}$ being the actual refractive index of the extraordinary ray and $n_{2}$ that of the ordinary ray.

The wave normal surfaces for the uniaxial crystals without optical activity have shapes of a sphere and an ellipsoid:

$$
\begin{gathered}
n_{20}(\theta)=n_{o}, \\
n_{10}(\theta)=\frac{n_{e} n_{o}}{\sqrt{n_{e}^{2} \cos ^{2} \theta+n_{o}^{2} \sin ^{2} \theta}},
\end{gathered}
$$

where $\theta$ means the angle between the directions of light propagation and the optic axis, and $n_{o}$ and $n_{e}$ the refractive indices of the ordinary and extraordinary rays peculiar for the direction perpendicular to the optic axis.

The gyration coefficient also depends on the direction and may be calculated from the gyration tensor components $g_{\mathrm{ij}}$ taking into account the symmetry of our crystal [6]:

$$
G(\theta)=g_{33} \cos ^{2} \theta+g_{11} \sin ^{2} \theta=g_{33}+\left(g_{11}-g_{33}\right) \sin ^{2} \theta .
$$

The gyration tensor component $g_{33}$ is easily obtained from the optical rotatory power $R$ along the optic axis:

$$
g_{33}=\frac{\lambda n_{0}}{\pi} R
$$

The dependences of the refractive indices $n_{o}$ and $n_{e}$ and the optical rotatory power $R$ upon the light wavelength $\lambda$ may be accurately approximated by a two-oscillator model [7]:

$$
\begin{aligned}
& n_{o}=\sqrt{1+\frac{F_{o}^{(1)}}{E_{o}^{(1) 2}-E(\lambda)^{2}}+\frac{F_{o}^{(2)}}{E_{o}^{(2) 2}-E(\lambda)^{2}}}, \\
& n_{e}=\sqrt{1+\frac{F_{e}^{(1)}}{E_{e}^{(1) 2}-E(\lambda)^{2}}+\frac{F_{e}^{(2)}}{E_{e}^{(2) 2}-E(\lambda)^{2}}},
\end{aligned}
$$




$$
R=S_{1}^{(1)}\left(\frac{E(\lambda)}{E_{1}^{(1) 2}-E(\lambda)^{2}}\right)^{2}+S_{1}^{(2)}\left(\frac{E(\lambda)}{E_{1}^{(2) 2}-E(\lambda)^{2}}\right)^{2}, E(\lambda)=\frac{h c}{e \lambda},
$$

where $h$ is the Planck constant, $c$ the free-space speed of light and $e$ the elementary charge. We have obtained the model parameters for the paratellurite after fitting the model to the experimental data reported in the study [7]:

$$
\begin{aligned}
& F_{o}^{(1)}=220.6 \mathrm{eV}^{2}, E_{o}^{(1)}=9.24 \mathrm{eV}, F_{o}^{(2)}=25.55 \mathrm{eV}^{2}, E_{o}^{(2)}=4.70 \mathrm{eV}, \\
& F_{e}^{(1)}=241.0 \mathrm{eV}^{2}, E_{e}^{(1)}=9.24 \mathrm{eV}, F_{e}^{(2)}=34.20 \mathrm{eV}^{2}, E_{e}^{(2)}=4.71 \mathrm{eV}, \\
& S_{1}^{(1)}=0.8838 \mathrm{rad} \times \mathrm{eV}^{2} / \mu \mathrm{m}, \mathrm{E}_{1}^{(1)}=9.31 \mathrm{eV}, \\
& S_{1}^{(2)}=0.08754 \mathrm{rad} \times \mathrm{eV}^{2} / \mu \mathrm{m}, \mathrm{E}_{1}^{(2)}=4.69 \mathrm{eV} .
\end{aligned}
$$

Using this model, we can find the $n_{o}, n_{e}$ and $g_{33}$ values for the wavelengths inside the range of $0.4-1.0 \mu \mathrm{m}$.

The transversal tensor component $g_{11}$ has not been established well up the present time. Only a few sources provide some estimations for the paratellurite, which vary significantly: $g_{11}=(-7.1 \pm 0.8) \times 10^{-3}[8]$ and $g_{11}=(-3.0 \pm 2.1) \times 10^{-4}[9]$ at $\lambda=632.8 \mathrm{~nm}$. However, our calculations show that the contribution of $g_{11}$ term to the resulting $n_{1}$ and $n_{2}$ values is negligibly small even for the largest reasonable term estimated as $g_{11}=-8 \times 10^{-3}$. Indeed, the difference does not exceed $0.001 \%$ for the whole $\theta$ range if we compare the actual refractive indices to those obtained while assuming that $g_{11}=0$.

As seen from Eq. (6), for the small $\theta$ values the major contribution to the gyration is caused by $g_{33}$. On the other hand, for the case of large angles $\theta$ the influence of birefringence component $\left(n_{20}^{2}-n_{10}^{2}\right)^{2}$ in Eqs. (2) and (3) overwhelms the optical activity contribution $4 G^{2}$. This greatly complicates obtaining a correct value of $g_{11}$ in any experiment and explains why the exact $g_{11}$ value has not yet been established.

\section{Sound wave propagation in paratellurite}

Dependence of the sound velocity of slow shear wave in the plane containing the directions [001] and [110] could be derived from the Christoffel equation in the following explicit form [10]:

$$
V_{s}\left(\theta_{A}\right)=\sqrt{\frac{1}{\rho}\left(\frac{c_{11}-c_{12}}{2} \cos ^{2} \theta_{A}+c_{44} \sin ^{2} \theta_{A}\right)},
$$

where $c_{11}=5.57 \times 10^{10} \mathrm{~N} / \mathrm{m}^{2}, c_{12}=5.12 \times 10^{10} \mathrm{~N} / \mathrm{m}^{2}$ and $c_{44}=2.65 \times 10^{10} \mathrm{~N} / \mathrm{m}^{2}$ are the stiffness tensor components for the paratellurite and $\rho=5990 \mathrm{~kg} / \mathrm{m}^{3}$ the density of the material. 
The difference between the directions of group and phase velocities may be easily derived from the velocity dependence [11]

$$
\tan \varphi=\frac{1}{V_{S}} \frac{\partial V_{S}}{\partial \theta_{A}}
$$

resulting in

$$
\varphi=\arctan \left(\frac{\left(2 c_{44}-c_{11}+c_{12}\right) \tan \theta_{A}}{c_{11}-c_{12}+2 c_{44} \tan \theta_{A}}\right) .
$$

Note that, according to the current definitions, the angle of the sound propagation $\theta_{A}$ and that of the optical dependence, $\theta$, are measured with respect to different axes.

\section{Calculations of interaction geometry}

Our deflector should enable controlling of diffracted beam by means of changing sound frequency. While doing the latter, we have the length of the sound wave vector $\mathbf{K}$ also changing and one can adjust the interaction geometry in order to change orientation parameters of the diffracted light. The magnitude and direction of the diffracted light wave vector $\mathbf{k}_{d}$ is constrained by angular dependence of the refractive index $n_{2}(\theta)$ for the ordinary ray. For satisfying the momentum-matching condition we should also adjust direction of the sound wave to make sure that the wave vectors add up properly. As said above, this small deviation in the sound direction is usually compensated by divergence of the sound wave.

To satisfy the Bragg condition inside a wide region of deflection angles, the angle $\theta_{d}$ describing the diffracted light should change in the region where the wave vector of sound is tangential to the wave normal surface $n_{2}(\theta)$ for the ordinary ray. Then only small deviation of the sound propagation angle from the original orientation will be required. In other words, the angle $\theta_{d}$ should change around some value $\theta_{\tau}$ corresponding to the spot where the sound vector touches the wave-normal surface tangentially. The angle $\theta_{\tau}$ for the tangential spot could be found after solving the equation

$$
\frac{\partial\left\{n_{2}\left(\theta_{\tau}\right) \sin \theta_{\tau}\right\}}{\partial \theta_{\tau}} \sin \theta_{A}+\frac{\partial\left\{n_{2}\left(\theta_{\tau}\right) \cos \theta_{\tau}\right\}}{\partial \theta_{\tau}} \cos \theta_{A}=0 .
$$

In principle, the implicit function involved in Eq. (14) may be inversed numerically, using any suitable method for finding function's roots (e.g., the Newton's method). However, numerical algorithms for calculating $\theta_{\tau}$ might be avoided owing to the fact that the wave-normal surface for the ordinary ray is almost a sphere. Deviation from a spherical shape is substantial only for the light propagation directions very close to the optic axis [001]. Taking into account that the sound wave is diverging, we have no need to find the exact value of the angle $\theta_{\tau}$ and may estimate it simply as $\theta_{\tau} \approx \theta_{A}$.

The angle $\theta_{d}$ of light deflection depends on the sound frequency $f_{S}$ and varies around the angle $\theta_{\tau}$. Then the angle $\theta_{\tau}$ may be considered as a diffraction angle at some 
"central" sound frequency $f_{0}$. All the construction parameters of deflectors are usually calculated using this "central" angle $\theta_{\tau}$, instead of true (varying) diffraction angle $\theta_{d}$.

We choose the angle of light incidence $\theta_{i}$ so that it made the diffracted light propagate at the angle $\theta_{\tau}$ after interaction. Simple geometric considerations show that there are two solutions for $\theta_{i}$. Writing down the expression for the tangent of $\theta_{A}$, one can find them from the equation

$$
\left(n_{1}\left(\theta_{i}\right) \sin \theta_{i}-n_{2}\left(\theta_{\tau}\right) \sin \theta_{\tau}\right) \sin \theta_{A}+\left(n_{1}\left(\theta_{i}\right) \cos \theta_{i}-n_{2}\left(\theta_{\tau}\right) \cos \theta_{\tau}\right) \cos \theta_{A}=0 .
$$

Solving Eq. (15) numerically for $\theta_{i}<\theta_{\tau}$ and $\theta_{i}>\theta_{\tau}$ gives us two values for the incidence angle, $\theta_{i}^{l}$ and $\theta_{i}^{h}$. To obtain diffraction at a specified angle $\theta_{\tau}$, we should direct the incident light at one or another angle. The difference is that the angle $\theta_{i}^{l}$ requires lower sound frequency, while the angle $\theta_{i}^{h}$ corresponds to higher frequency. Furthermore, the signs of the optical-frequency shift are opposite for these cases.

For a fixed incident angle $\theta_{i}$, the diffraction angle $\theta_{d}$ could differ from the value $\theta_{\tau}$ guessed initially. The actual diffraction angle $\theta_{d}$ depends on the sound frequency $f_{S}$ and this just enables operation of the device as a laser beam deflector. The sound frequency and the desired deflection angle are related as

$$
\begin{aligned}
& f_{S}\left(\theta_{i}, \theta_{d}\right)=\frac{V_{S}\left(\theta_{S}\right)}{\lambda} \sqrt{n_{1}\left(\theta_{i}\right)^{2}+n_{2}\left(\theta_{d}\right)^{2}-2 n_{1}\left(\theta_{i}\right) n_{2}\left(\theta_{d}\right) \cos \left(\theta_{i}-\theta_{d}\right)}, \\
& \theta_{S}\left(\theta_{i}, \theta_{d}\right)=-\arctan \left(\frac{n_{1}\left(\theta_{i}\right) \cos \theta_{i}-n_{2}\left(\theta_{d}\right) \cos \theta_{d}}{n_{1}\left(\theta_{i}\right) \sin \theta_{i}-n_{2}\left(\theta_{d}\right) \sin \theta_{d}}\right) .
\end{aligned}
$$

Here $\theta_{S}$ represents the angle of sound wave propagation required for the case of given $\theta_{i}$ and $\theta_{d}$. The angle $\theta_{S}$ needed differs slightly from the sound-beam orientation angle $\theta_{A}$, but the diffraction still occurs because of angular divergence of the sound. Using tangential spot $\theta_{\tau}$ as an initial approximation for the diffraction angle $\theta_{d}$ ensures that the difference between $\theta_{S}$ and $\theta_{A}$ would be minimal for a wide range of deflection angles.

The available range of diffraction angles depends on angular distribution of the sound intensity. The angular spectrum of sound radiated by rectangular transducer may be expressed as a square of function $\operatorname{sinc}(x)=\sin (\pi x) / \pi x$. The angular distribution of the sound power [12] is determined by

$$
P_{A}\left(\delta \theta_{S}\right)=P_{0} \operatorname{sinc}^{2}\left(\frac{L f_{S}}{V_{S}} \delta \theta_{S}\right),
$$

where $\delta \theta_{S}=\theta_{S}-\theta_{A}$ is the angle of deviation from the direction of sound propagation $\theta_{A}, f_{S}$ the sound frequency and $L$ the length of the transducer. The first zeros of the 
function given by Eq. (18) correspond to angular width of the principal lobe of radiation pattern.

The efficiency of the light diffraction depends directly on the sound intensity. Therefore the available deflection range may be estimated from the zeroes of sound power distribution function. It should be taken into account that the sound radiation pattern depends on the sound frequency. Moreover, it should also be noted that only half of the sound radiation pattern participates in the diffraction. For the smaller and larger light incident angles (i.e., $\theta_{i}^{l}$ and $\theta_{i}^{h}$ ) these are parts with the positive and negative $\delta \theta_{S}$ values, respectively. The minimal and maximal deflection angles may be found from zeros of the sinc function by setting its argument to be unit:

$$
\frac{L f_{S}\left(\theta_{i}^{l}, \theta_{d}\right)}{V_{S}\left(\theta_{A}\right)}\left(\theta_{S}\left(\theta_{i}^{l}, \theta_{d}\right)-\theta_{A}\right)=1
$$

Solving Eq. (19) numerically would give us some angles $\theta_{d \min }^{l}$ and $\theta_{d \max }^{l}$ for the lower-frequency version of the device. When finding the same angles for the higherfrequency version, Eq. (19) should be replaced by

$$
\frac{L f_{S}\left(\theta_{i}^{h}, \theta_{d}\right)}{V_{S}\left(\theta_{A}\right)}\left(\theta_{S}\left(\theta_{i}^{h}, \theta_{d}\right)-\theta_{A}\right)=-1 \text {. }
$$

Again, solving Eq. (20) would yield in parameters $\theta_{d \min }^{h}$ and $\theta_{d \max }^{h}$ searched for. The corresponding region for the driving frequency $f_{S}$ may be estimated from the angles obtained using Eqs. (16) and (17).

\section{Two-wavelength operation of deflector}

The angles $\theta_{i}$ and $\theta_{d}$ are internal angles and describe light propagation inside the crystal. The external angles of the input and output beams for a real device are determined by inclination of the input and output faces of acoustooptic cell. The input and output beams should be collinear at the central frequency. To ensure this, we set the output face to be normal to the diffracted light direction at the central angle $\theta_{\tau}$. The inclination angle $\beta$ of the input face is to be set such that the refracted beam propagate inside the crystal exactly at the angle $\theta_{i}$. Using the Snell's law, we have

$$
\beta=\arctan \left(\frac{\sin \left(\theta_{A}-\theta_{i}\right)}{\cos \left(\theta_{A}-\theta_{i}\right)-n_{3} / n_{1}\left(\theta_{i}\right)}\right),
$$

where $n_{3}$ is the refractive index of surrounding media (for the air we assume $n_{3}=1$ ).

In the general case, the angle $\beta$ depends on the light wavelength. Using relationships given above we have obtained the $\beta_{1}$ and $\beta_{2}$ values for the wavelengths of $\lambda_{1}=632.8 \mathrm{~nm}$ and $\lambda_{2}=785 \mathrm{~nm}$. The calculations show that the $\beta_{1}^{l}$ and $\beta_{2}^{l}$ values are very close to each other. The angles $\beta_{1}^{h}$ and $\beta_{2}^{h}$ associated with the alternative geometry 
of diffraction are also almost the same (see Fig. 4). Because of small difference of $\beta$ 's for the two wavelengths, the average value $\beta_{m}=\left(\beta_{1}+\beta_{2}\right) / 2$ can be used when constructing a dual-wavelength device. In this case, there will be small detuning of the sound orientation at the both wavelengths,

$$
\Delta \theta_{S 1}=\theta_{S}\left(\theta_{i 1}, \theta_{\tau}\right)-\theta_{A}, \Delta \theta_{S 2}=\theta_{S}\left(\theta_{i 2}, \theta_{\tau}\right)-\theta_{A},
$$

where the angles $\theta_{i 1}$ and $\theta_{i 2}$ of light incidence differ for different wavelengths. The internal angles $\theta_{i 1}, \theta_{i 2}$ depend on how light enters the crystal and they are determined by the input face inclination. According to the Snell's law, we get

$$
n_{3} \sin \beta=n_{1}\left(\lambda, \theta_{i}\right) \sin \left(\theta_{i}-\theta_{A}+\beta\right) \text {. }
$$

Solving this implicit equation numerically for $\theta_{i}$, we will find the actual angles $\theta_{i 1}$ and $\theta_{i 2}$ for the incident light at the both wavelengths for a given input face orientation $\beta$.
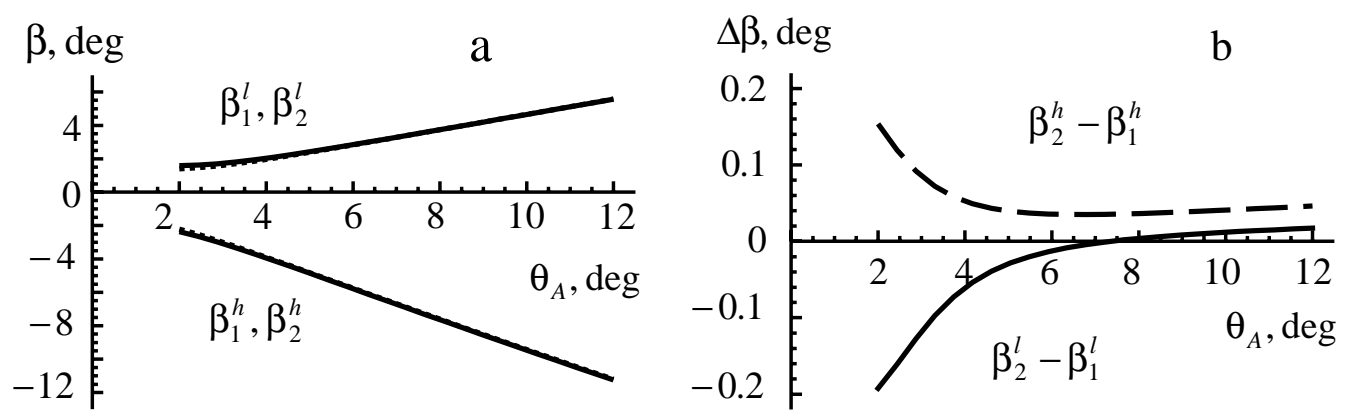

Fig. 4. Dependences of input face inclination angles on the sound propagation direction for the two different light wavelengths and both lower- and higher-frequency interaction geometries (a) and the corresponding plots for the angular differences (b). The latter plots are presented separately since the angles for different wavelengths are close.

Plots of the sound detuning $\Delta \theta_{S 1}^{l}, \Delta \theta_{S 2}^{l}$ and $\Delta \theta_{S 1}^{h}, \Delta \theta_{S 2}^{h}$ obtained for the two wavelengths and the both possible diffraction geometries are presented in Fig. 5. Typically the angle of sound divergence $\delta \theta_{A}$ is much larger than the detuning angle $\Delta \theta_{S}$ (cf. Fig. 5 and Fig. 6). Then the two-wavelength operation should be possible almost for all the sound orientations $\theta_{A}$ whenever the input face inclination angle is chosen as suggested above.

To obtain a wide range of deflection angles, some divergence of the sound is needed. The minimal and maximal deflection angles are determined by the sound radiation pattern. The maximal deviation of the sound wave vector $\delta \theta_{S}=\theta_{S}-\theta_{A}$ required for obtaining full range of deflection is shown in Fig. 6. The plots in Fig. 6 have been calculated from the relations for the boundary deflection angles $\theta_{d \text { min }}$ and $\theta_{d \max }$, with assuming 
that the transducer's length is $L=2.5 \mathrm{~mm}$. It is seen that the range of sound wave vector deviation needed for operation of the deflector is much larger than the detuning $\Delta \theta_{S 1}$ and $\Delta \theta_{S 1}$ caused by inexact orientation of the input face $\left(\beta_{m}=\left(\beta_{1}+\beta_{2}\right) / 2\right)$. It is this fact that enables us to construct the two-wavelength deflector.

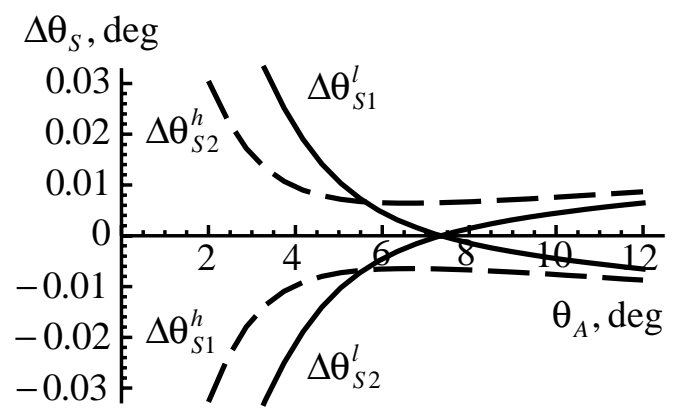

Fig. 5. Sound detuning caused by inexact orientation of the input face as a function of sound propagation direction.

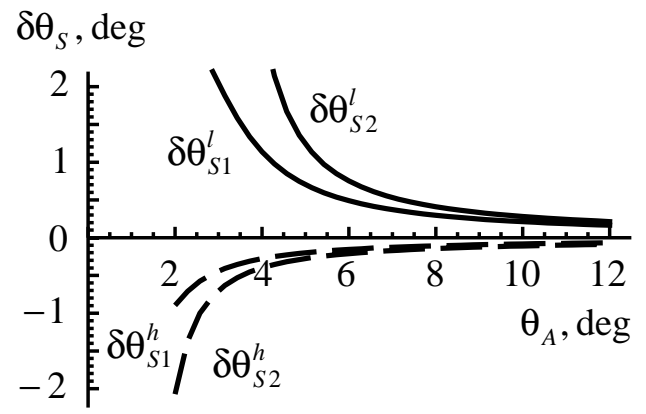

Fig. 6. Sound deviation needed for operation of the deflector as a function of sound propagation direction.

The diffracted light is refracted at the output face of the crystal and therefore the deflection angle is also affected by dispersion of the refractive index. The external angle of the deflected beam after passing the output face may be expressed as

$$
\theta_{x}=\arcsin \left(\frac{n_{2}\left(\theta_{d}\right)}{n_{3}} \sin \left(\theta_{d}-\theta_{A}\right)\right)
$$

The ranges of deflection angles available are slightly different for the wavelengths used (see Fig. 7), the higher wavelength producing larger deflection range.

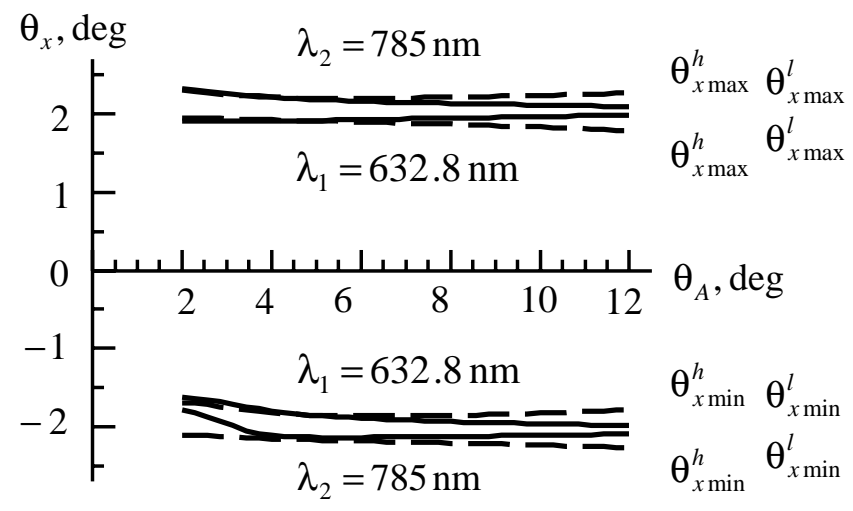

Fig. 7. Minimal and maximal deflection angles found from zeroes of the sound radiation pattern as functions of sound propagation direction.

Since the angular range is estimated by the zeroes of the sound distribution function, the useful deflection range (characterised by high enough diffraction efficiency) would be nearly twice smaller than that depicted in Fig. 7. Interesting enough is the fact that the available deflection range almost does not depend on the sound orientation angle $\theta_{A}$, if only the size $L$ of the transducer remains fixed. 
The frequency range corresponding to deflection from the minimal angle to the maximal one depends upon the light wavelength and the sound orientation angle $\theta_{A}$. The $\theta_{x}$ parameters as functions of the sound frequency are depicted in Fig. 8 for the two light wavelengths and different $\theta_{A}$. It is seen from Fig. 8 that there are regions with the same deflection angle for different light wavelengths, which require different driving frequencies. By choosing two non-overlapping frequency regions, we obtain the same deflection range at the both wavelengths and simultaneously control appearing of one or another light wavelength by changing the sound frequency.

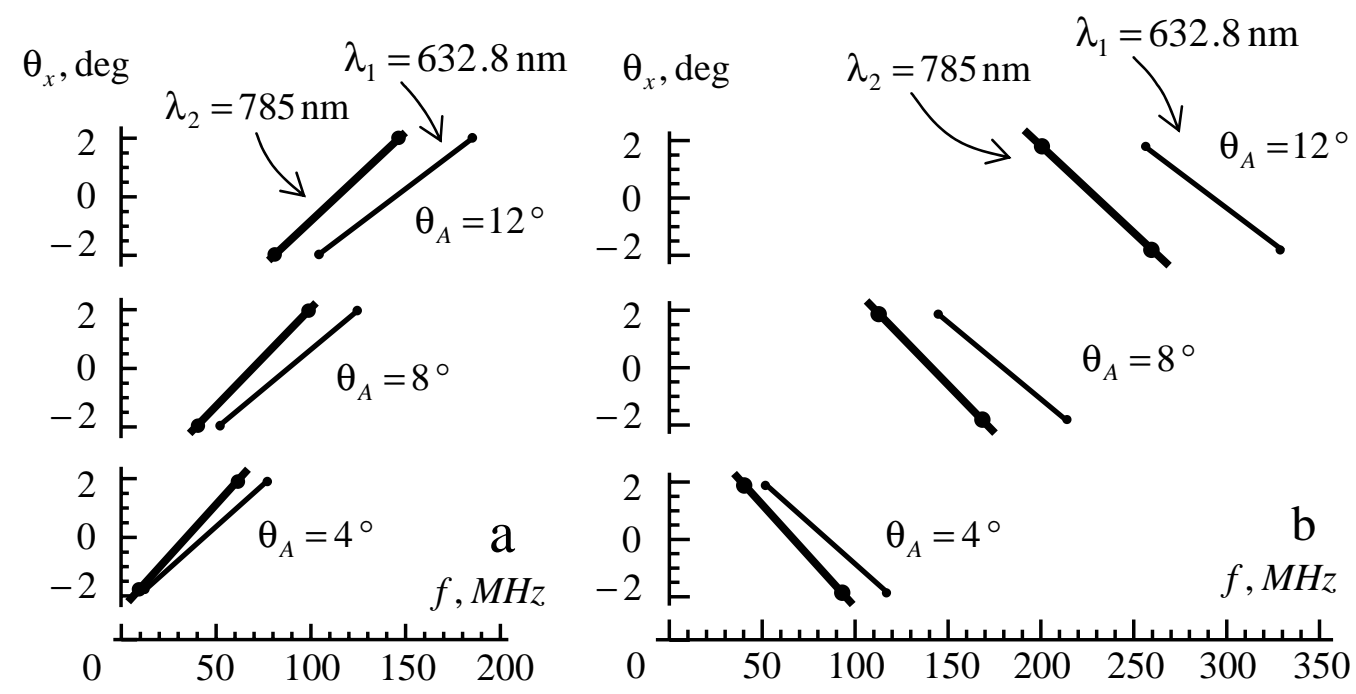

Fig. 8. Dependences of output angle on the driving frequency for several sound parameters corresponding respectively to lower- (a) and higher-frequency (b) versions of the device.

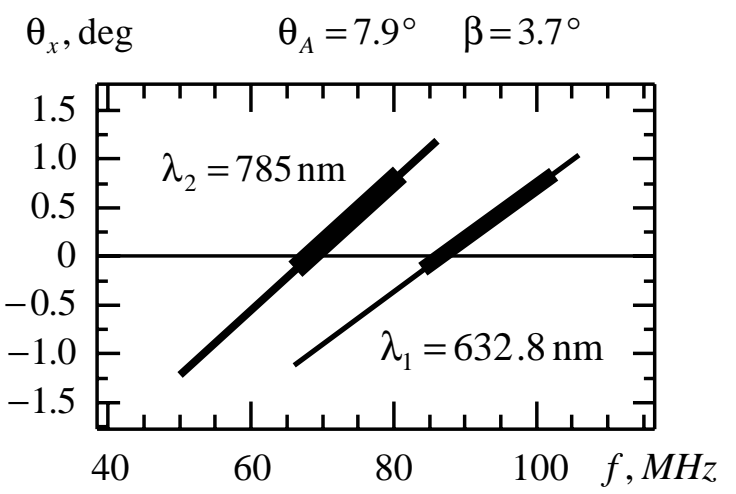

Fig. 9. Deflection angle as a function of driving frequency. Two non-overlapping frequency regions with the same output angular ranges are highlighted.

In order to verify the proposed construction experimentally, we have manufactured the actual device, using the working parameters $\theta_{A}=7.9^{\circ}, \beta=3.7^{\circ}, L=2.5 \mathrm{~mm}$ and $H=8 \mathrm{~mm}$. The resulting angular response of deflection for these parameters is presented in Fig. 9. By changing the frequency from 65 up to $80 \mathrm{MHz}$, we have obtained the 
light diffraction on the larger wavelength $\left(\lambda_{2}=785 \mathrm{~nm}\right)$. The frequency changes in the region of $85-105 \mathrm{MHz}$ have resulted in the diffraction on the smaller wavelength $\left(\lambda_{1}=632.8 \mathrm{~nm}\right)$, with the same angular deflection range. In this manner the wavelength switching has been realised in the device, along with the light deflection. At some frequencies, efficient enough diffraction is possible at the both wavelengths, though the deflection angle is outside a desired range. While eliminating the light of unneeded wavelength, we have still to use a diaphragm.

To avoid the appearance of both wavelengths at the same time, another approach could be used. The full deflection range could be made smaller by increasing the transducer's length. This should narrow the sound radiation pattern and separate the working frequency ranges, so that there would be no diffraction at the other wavelength of light.

\section{Diffraction efficiency}

The actual working range of the deflector is determined by the region where the diffraction efficiency is high enough for practical utilisation. The efficiency of acoustooptic interaction is determined by photoelastic properties of the material. Taking into account angular changes in the effective photoelastic constant [13] and elongation of the interaction length due to oblique incidence of the light on the sound beam [13], one can express the diffracted light intensity as

$$
I=I_{0} \sin ^{2} \eta
$$

where

$$
\eta=\frac{\pi}{\lambda} \frac{\cos \varphi}{\cos \left(\theta_{A}-\theta_{i}+\varphi\right)} \sqrt{\frac{P_{A} L}{2 H}\left[\frac{V_{S}\left(\theta_{A}\right)}{V_{S}(0)}\right]^{3} M_{2} \cos ^{2} \theta_{i}} .
$$

Here $M_{2}=n^{6} p^{2} / \rho V_{S}^{3}$ is the figure of merit at $\theta_{A}=0^{\circ}$ and $\theta_{i}=0^{\circ}, \varphi$ the angle between the phase and group velocity vectors of the sound, $P$ the sound power, $L$ the transducer's length, and $H$ the transducer's width in the perpendicular direction. The tabulated value $M_{2}=793 \times 10^{-15} \mathrm{~s}^{3} / \mathrm{kg}$ may be found in [14].
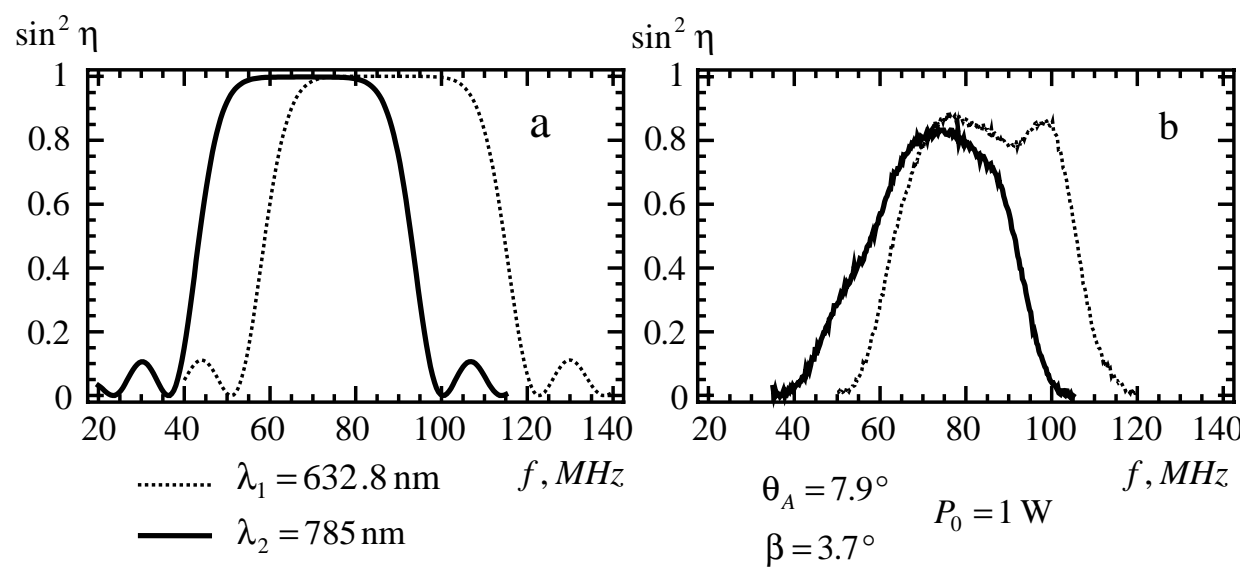

Fig. 10. Calculated (a) and experimentally measured (b) diffraction efficiencies for the two different light wavelengths. 
The dependences of calculated and measured diffraction efficiencies on the driving frequency for the configuration selected above are presented in Fig. 10. When compare to the theoretical dependence, the experimental one involves a noticeable decrease in the efficiency at lower frequencies, which reduces performance of the device on the second light wavelength. This is caused by inherent band shape of the ultrasonic transducer, which has been designed for the central frequency of $85 \mathrm{MHz}$ and has the bandwidth of $40 \mathrm{MHz}$. The efficiency drop could be partially compensated owing to increasing driving power of the transducer at lower frequencies. In general, any dual-wavelength operation requires transducers to be matched for broader frequency range, contrary to singlewavelength devices. This complicates both the transducer construction and the matching circuits. In some cases special wide-band transducer matching [15] should be used in order to obtain decent efficiency at the both wavelengths.

\section{Conclusions}

An acoustooptic deflector operating at two different wavelengths of light has been suggested. The device is constructed so that the diffraction conditions are satisfied at the both wavelengths simultaneously. Analysis of the conditions under which the two-wavelength operation is possible has been given. A complete procedure for calculating the device parameters has also been presented. The device could perform beam scanning over the same grid of points at the two light wavelengths. At the same time, it is capable of switching between those wavelengths. The working wavelength may be selected by changing the driving frequency, with no mechanical readjustment.

Two-wavelength operation has imposed some limitations, if compare to its single-wavelength analogue. If the working wavelengths are close to each other, a full separation of frequency bands is impossible, so that any undesired light outside the working range of angles should be eliminated by a diaphragm. Moreover, the angular deflection range available for the two-wavelength operation is smaller than that for the single-wavelength case, although here the same device performs switching of the wavelength. If laser beams are switched on-off by some other means, the deflector is capable of operating in the full angular range. The frequency band of the ultrasonic transducer needed for the two-wavelength operation is broader than that for the single-wavelength device.

Experimental verification has confirmed a possibility for constructing this dualwavelength deflector. Some degradation of the deflector's performance observed in the experiment at low sound frequencies is due to limited band shape of the ultrasonic transducer. Nonetheless, the device constructed by us has its advantage in combining deflection ability with a wavelength-switching one. The diffraction efficiency achieved with our device is as high as that for single-wavelength devices. It is approximately equal to 80 per cent for the both wavelengths within the angular deflection range used.

The considerations presented in this work may be used not only when constructing acoustooptic devices that operate at different wavelengths but also for analysing 
off-the-shelf products in the conditions when one needs to change the working wavelength. Let us finally make the only example. It is known that the angular dispersion may be accurately determined if the input light has a wide spectrum, due to ultra-short pulse duration. This angular broadening of the diffracted beam has already been observed in the experiments [16] and, what is more, the current results could be useful when constructing the corresponding compensation system.

\section{References}

1. Gazalet M G, Bruneel C, Waxin G, Rouvaen J M and Bridoux E, 1985. Tunable bichromatic acoustooptic deflector. Appl. Opt. 24: 243-246.

2. Kotov V M, 1995. Acoustooptic modulation of multicomponent Ar laser radiation. Quantum Electronics. 25: 552-555.

3. Kotov V M, 1997. $\mathrm{TeO}_{2}$ single-crystal acoustooptic $2 \times 2$ switch for light beams of different wavelengths. Techn. Phys. 42: 181-185.

4. Kim S, Mcleod R R, Saffman M and Wagner K H, 2008. Doppler-free, multiwavelength acoustooptic deflector for two-photon addressing arrays of $\mathrm{Rb}$ atoms in a quantum information processor. Appl. Opt. 47: 1816-1831.

5. Yano T, Kawabuchi M, Fukumoto A and Watanabe A, 1975. $\mathrm{TeO}_{2}$ anisotropic Bragg light deflector without midband degeneracy. Appl. Phys. Lett. 26: 689-691.

6. Yariv A and Yeh P, Optical waves in crystals. New York: A Wiley-Interscience Publ. (1987).

7. Uchida N, 1971. Optical properties of single-crystal paratellurite $\left(\mathrm{TeO}_{2}\right)$. Phys. Rev. B. 4: $3736-3745$.

8. Vlokh R, 2001. Crystallooptics of the circularly polarized waves. Ukr. J. Phys. Opt. 2: 107-125.

9. Mamedov N, Yamamoto N, Shim Yo, Ninomiya Yu and Takizawa T, 2003. Extended application of light figures to optically active materials with transversally isotropic dielectric function. Japan. J. Appl. Phys. 42: 5145-5152.

10. Bogdanov S.V., Technique for calculating an acoustooptic deflector on paratellurite. In the book: Acoustooptic devices of radioelectronic systems. Leningrad: Nauka (1988).

11. Ohmachi Yo, Uchida $\mathrm{N}$ and Niizeki N, 1972. Acoustic wave propagation in $\mathrm{TeO}_{2}$ single crystal. J. Acoust. Soc. Amer. 51: 164-168.

12. Gordon E I, 1966. A review of acoustooptical deflection and modulation devices. Appl. Opt. 5: 1629-1639.

13. Yano $\mathrm{T}$ and Watanabe A, 1976. Acoustooptic $\mathrm{TeO}_{2}$ tunable filter using far-off-axis anisotropic Bragg diffraction. Appl. Opt. 15: 2250-2258.

14. Uchida $\mathrm{N}$ and Ohmachi Yo, 1969. Elastic and photoelastic properties of $\mathrm{TeO}_{2}$ single crystal. J. Appl. Phys. 40: 4692-4695.

15. Yurchenko A V, 1995. Wide-band transformerless matching of high-frequency piezotransducers. Izv. Vyssh. Uchebn. Zaved. Radioelektronika. 7: 41-48. 
16. Ngoi B K A, Venkatakrishnan K, Tan B, Stanley P and Lim L E N, 2001. Angular dispersion compensation for acoustooptic devices used for ultrashort-pulsed laser micromachining. Opt. Express. 9: 200-206.

Pilgun Yu.V. and Smirnov Ye.M., 2010. Broadband acoustooptic diffraction of two-wavelength light in paratellurite. Ukr.J.Phys.Opt. 11: 28-43.

Анотація. Запропоновано акустооптичний дефлектор, щуо працює на двох довжинах хвиль світла. Геометрію кристала парателуриту обрано так, щуоб одночасно задовольнити умови Брелта на обох довжинах хвиль. Це дає змогу отримати відхилення світла тої чи іншої довжини хвилі в однаковому кутовому діапазоні. Перемикання між довжинами хвиль відбувається за рахунок зміни частоти звуку, тому в певний момент часу на виходi дефлектора присутнє світло лише однієї довжини хвилі. У роботі представлено метод розрахунку параметрів такого пристрою. Відповідний дефлектор виготовлено та досліджено експериментально. 